\title{
Metástasis hepáticas en el cáncer colorrectal: estrategias terapéuticas y recomendaciones actuales
}

\author{
Diego Dávila ${ }^{1}$, Óscar Palacios ${ }^{1}$, Camilo Naranjo $^{2}$
}

Palabras clave: neoplasias; hígado; neoplasias del colon; metástasis de la neoplasia; neoplasias primarias secundarias; hepatectomía; protocolos clínicos; supervivencia.

\section{Resumen}

El cáncer colorrectal con metástasis a higado es una enfermedad frecuente que hasta hace pocos años se consideraba únicamente de tratamiento paliativo. Sin embargo, con la reciente aparición de nuevas técnicas $y$ avances para aumentar la posibilidad de resecar las lesiones y los nuevos esquemas de quimioterapia, se ha logrado la resección quirúrgica de este tipo de lesiones, extrayendo todo el tejido tumoral macroscópico e impactando de forma positiva la supervivencia de estos pacientes.

Múltiples esquemas se han propuesto para el tratamiento de las lesiones sincrónicas con el fin de mejorar la supervivencia de los pacientes. Si bien no hay consenso sobre cuál es el mejor esquema terapéutico en estos casos, parece ser que lo más importante es que se logre completar de manera exitosa el esquema elegido. En general, la tendencia actual es practicar las intervenciones quirúrgicas del hígado primero, a menos

1 Médico, cirujano general, subespecialista en Cirugía HepatoPancreato-Biliar, Universidad CES, Medellín, Colombia

2 Médico, residente de Cirugía General, Universidad CES, Medellín, Colombia

Fecha de recibido: 8 de junio de 2017

Fecha de aprobación: 19 de julio de 2017

Citar como: Dávila D, Palacios Ó, Naranjo C. Metástasis hepáticas en el cáncer colorrectal: estrategias terapéuticas y recomendaciones actuales. Rev Colomb Cir. 2017;32:304-18. que el tumor primario se encuentre sintomático; en este caso, la colectomía es el abordaje inicial. Las resecciones simultáneas hacen parte del arsenal terapéutico de estos pacientes. Múltiples grupos sugieren no asociar una resección de recto bajo a una hepatectomía mayor. Por su parte, las lesiones metacrónicas deben resecarse según el tamaño y la posibilidad de resección una vez hecho el diagnóstico.

Es importante tener en cuenta que, aunque se logre una resección completa del tejido tumoral con márgenes $R_{0}$, las recurrencias son frecuentes debido a que la diseminación tumoral microscópica ya está presente; por esta razón, es indispensable agregar la quimioterapia para mejorar el pronóstico de los pacientes.

\section{Introducción}

El cáncer colorrectal es la tercera neoplasia más frecuente a nivel mundial tanto en hombres como en mujeres. Se estima que para finales del 2017 se presentarán 135.430 casos nuevos solo en Norteamérica ${ }^{1}$. Si bien la mortalidad por cáncer de colon ha disminuido el $51 \%$ con respecto a 1976, aún continúa siendo una importante causa de mortalidad ${ }^{2,3}$ y corresponde a la segunda causa de muerte por cáncer en los hombres y la tercera en las mujeres. El hígado es su principal sitio de metástasis, debido a que el drenaje venoso del colon confluye a este órgano ${ }^{4}$. Al momento del diagnóstico, el $25 \%$ de los pacientes con cáncer colorrectal presentan metástasis hepáticas ${ }^{5,6}$ y la tercera parte de los que no, la desarrollará en los tres años siguientes ${ }^{7,8}$. 
La supervivencia a cinco años de los pacientes con cáncer colorrectal con metástasis a hígado es de menos del $10 \%$, con una tasa de curación menor del $2 \%$ con los esquemas terapéuticos paliativos disponibles, mientras que, cuando se logra practicar una resección oncológica reglada, con márgenes negativos para malignidad, tanto de las metástasis como de la lesión tumoral primaria, la supervivencia a más de $67 \%$, con una tasa de curación del 15 al $30 \%{ }^{9}$.

Por esta razón, se afirma que la cirugía es el único tratamiento que impacta en la supervivencia a largo plazo y, en la actualidad, el esfuerzo terapéutico está dirigido a aumentar el número de candidatos a la resección quirúrgica, ya que solo 15 a $20 \%$ de estos tumores puede resecarse cuando se hace el diagnóstico ${ }^{10-13}$. Actualmente, existen múltiples estrategias que permiten convertir los casos no aptos para resección en candidatos al manejo quirúrgico, con un impacto positivo en el pronóstico oncológico. Sin embargo, está claro que, a pesar de que se logre hacer una resección completa de las lesiones, la recurrencia se presenta en 60 a $65 \%$ de los casos ${ }^{14-18} \mathrm{y}$, de ahí, la importancia de adicionar esquemas de quimioterapia sistémica, con el fin de mejorar el pronóstico.

\section{Lesiones metastásicas sincrónicas $V s$. metacrónicas}

Las metástasis sincrónicas son aquellas que se diagnostican al mismo tiempo que la lesión tumoral primaria. Las metástasis metacrónicas son aquellas que se descubren después del diagnóstico o de la resección del tumor primario; estas se clasifican como tempranas o tardías, cuando aparecen antes de los 12 meses o después, respectivamente. Las lesiones sincrónicas tienen un peor pronóstico oncológico que las metacrónicas, debido a que son biológicamente más agresivas $\mathrm{y}$, por ende, su comportamiento tumoral es más impredecible ${ }^{19,20}$. Esto plantea un gran reto terapéutico para el cirujano, no solo por lo anteriormente expuesto, sino también por el hecho de que en estos pacientes se encuentra asociada la lesión tumoral primaria, la cual también se debe resecar lo más pronto posible.

\section{Clasificación}

Por definición, la enfermedad colorrectal con metástasis a hígado se ha considerado siempre como un estadio
IV y, como históricamente solo del 15 al $20 \%$ de los pacientes en este estadio se podían someter directamente a cirugía con intención curativa, la inmensa mayoría recibía únicamente tratamientos paliativos.

No obstante, con los nuevos esquemas de quimioterapia, se comenzó a observar una gran reacción sistémica en las metástasis hepáticas y, actualmente, se sabe que de 30 a $35 \%$ de las metástasis que no se pueden resecar cuando se hace el diagnóstico, mejoran tanto con la quimioterapia que, después de esta, pueden intervenirse quirúrgicamente con fines curativos. Por esta razón, hoy en día, para establecer que un paciente con cáncer de colon o recto y metástasis hepáticas solo puede recibir tratamiento paliativo, se requiere administrar un ciclo completo de quimioterapia y, después, reevaluar las lesiones; si nuevamente se considera que no pueden resecarse, el tratamiento se limita a la paliación ${ }^{21}$.

El European Colorectal Metastases Treatment Group propone clasificar en cuatro grupos a los pacientes con cáncer colorrectal, según las metástasis hepáticas: $\mathrm{M}_{0}$, $\mathrm{M}_{1 \mathrm{a}}, \mathrm{M}_{1 \mathrm{~b}}$ y $\mathrm{M}_{1 \mathrm{c}}$. En $\mathrm{M}_{0}$, no hay metástasis; en $\mathrm{M}_{1 \mathrm{a}}$, existen metástasis hepáticas resecables que se van a beneficiar de una resección quirúrgica temprana con el fin de evitar su progresión; en $\mathrm{M}_{1 \mathrm{~b}}$, hay metástasis hepáticas potencialmente resecables: es necesario el tratamiento previo para su resección, bien sea quimioterapia neoadyuvante o métodos de hipertrofia hepática; en $\mathrm{M}_{1 \mathrm{c}}$, hay metástasis hepáticas con casi ninguna posibilidad de resección; solo se pueden clasificar así ante la ausencia de mejoría con un ciclo completo de quimioterapia y solo se beneficiarán de un tratamiento paliativo (figura1) ${ }^{22}$.

\section{Criterios para resección}

Uno de los temas de mayor variación en los últimos años, y uno de los que más debate ha generado entre los cirujanos y que al mismo tiempo más ha revolucionado la cirugía hepatobiliar, ha sido establecer cuáles pacientes con lesiones neoplásicas hepáticas pueden someterse a resección quirúrgica con un adecuado pronóstico oncológico y de manera segura.

Tradicionalmente, las metástasis en hígado se han considerado difíciles de resecar y, solo bajo unas condiciones específicas, se beneficiaban de la resección quirúrgica. Hace algunos años, únicamente los pacientes 


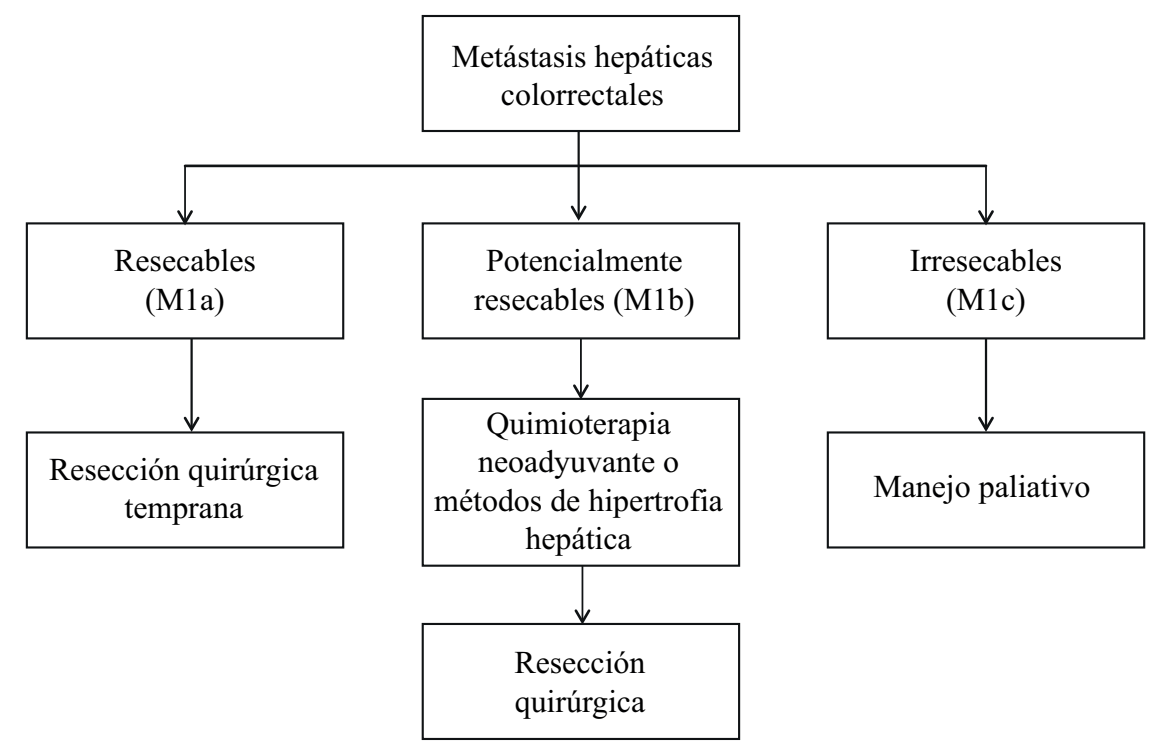

FIGURA 1. Clasificación de las metástasis hepáticas de origen colorrectal

con menos de tres metástasis metacrónicas, unilobulares y de menos de cinco centímetros de diámetro, eran candidatos a la resección quirúrgica con fines curativos; estos pacientes solo representaban el $10 \%$ de todos los casos con compromiso hepático secundario a la diseminación de células tumorales provenientes de colon y recto ${ }^{23}$.

Sin embargo, recientemente se ha venido discutiendo si solo los pacientes con estas características son candidatos a resección quirúrgica con fines curativos o si, por el contrario, es posible practicarla en otras situaciones, garantizando los mismos resultados en quienes inicialmente no eran candidatos por no cumplir con los criterios clásicos de resección.

En el 2006, hubo un cambio importante en el entendimiento de este tipo de lesiones y en su enfoque. Se dejó de considerar a las metástasis como el factor determinante de la posibilidad de resección y se dio más importancia al parénquima hepático sano residual posquirúrgico, lo cual generó nuevos criterios de selección, con los cuales se aumentó el porcentaje de pacientes con posibilidad de resección oncológica (más del $30 \%$ en la actualidad) y con mejores resultados en términos de complicaciones y morbimortalidad posoperatoria.

En el 2013, se llevó a cabo un consenso de expertos en el que se determinó con claridad cuáles eran los requisitos para catalogar a un paciente con metástasis hepáticas de origen colorrectal como candidato a resección quirúrgica ${ }^{24}$. Se concluyó que, inicialmente, se debe hacer una búsqueda exhaustiva de enfermedad metastásica extrahepática; en caso de existir, debe ser factible su resección quirúrgica o su tratamiento con quimioterapia o con técnicas ablativas (criterio oncológico). Una vez cumplido este primer requisito, es necesario establecer si la lesión neoplásica primaria es técnica o anatómicamente resecable (criterio anatómico).

De esta manera, se consideran como criterios de resección: garantizar una extracción de la pieza quirúrgica con márgenes microscópicamente sanos (resección $\mathrm{R}_{0}$ ), preservar un adecuado remanente hepático funcional y respetar los principios quirúrgicos de la cirugía hepática tradicional (tabla 1). Por lo tanto, las metástasis colorrectales solo se consideran irresecables cuando anatómicamente es imposible su extracción por el compromiso vascular que presentan o cuando el hígado remanente es de un volumen menor del 20 al $30 \%$ del total, según el paciente, $y$, por ende, hay un mayor riesgo de falla hepática (figura 2).

\section{Resección quirúrgica}

El hígado es un órgano complejo y, por ende, los procedimientos quirúrgicos hepáticos requieren no solo de un 
TABLA 1.

Criterios de resección de las metástasis hepáticas

\section{Criterios oncológicos}

1. Estadificación radiológica en búsqueda de enfermedad extrahepática

2. Enfermedad metastásica extrahepática resecable

3. Garantizar respuesta oncológica de la lesión a la quimioterapia

\section{Criterios anatómicos}

1. Garantizar una resección R0

2. Adecuado volumen residual funcional hepático

3. Respetar los principios quirúrgicos de la cirugía hepática tradicional

a. Garantizar un flujo de salida (una vena suprahepática)

b. Garantizar un flujo de entrada (porta, arteria hepatica)

c. Garantizar un drenaje biliar

d. Dejar, al menos, dos segmentos hepáticos contiguos

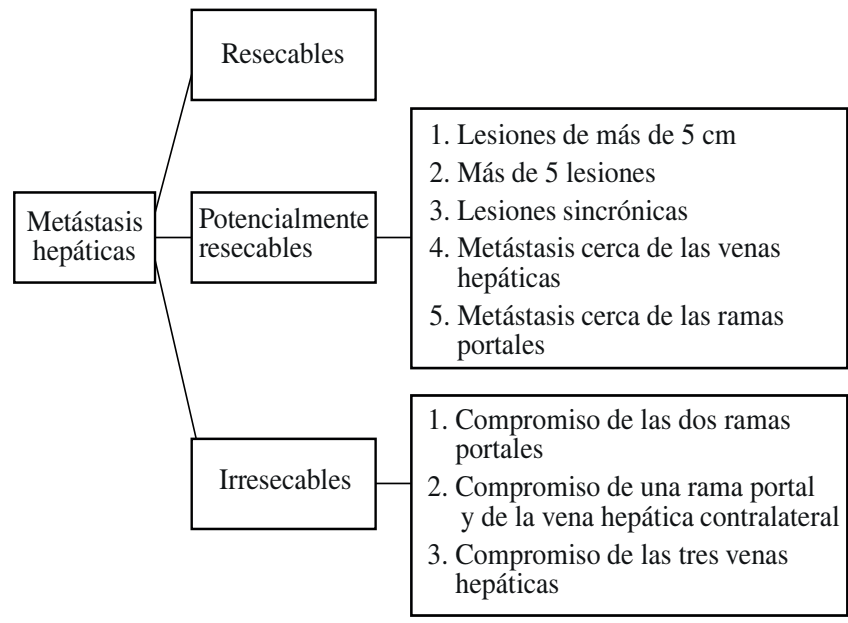

Figura 2. Clasificación de las metástasis hepáticas en cáncer colorrectal

conocimiento preciso de la anatomía, sino también, de una técnica quirúrgica depurada y precisa que permita la resección completa de las lesiones neoplásicas, dejando siempre un adecuado parénquima funcional que sea compatible con la vida ${ }^{25,26}$.

Los objetivos quirúrgicos son poca pérdida sanguínea, periodos cortos de isquemia hepática y baja morbimortalidad posquirúrgica. La pérdida sanguínea es por sí misma un factor predictor independiente de mortalidad e impacta, al igual que las complicaciones posoperatorias, en el pronóstico a largo plazo de las metastasectomías hepáticas por cáncer colorrectal.
Es necesario garantizar un volumen mínimo de hígado remanente, adecuado para un buen funcionamiento que evite la aparición de falla hepática posquirúrgica y la muerte. En general, se acepta que en los pacientes oncológicos se deje un volumen residual funcional de más del $20 \%$, cuando el parénquima hepático se considera sano, de más del $30 \%$, cuando ha habido quimioterapia previa, y de más del $40 \%$, cuando existe alguna hepatopatía crónica ${ }^{27,28}$.

Idealmente, a menos que sea indispensable, se debe evitar la maniobra de Pringle (pinzar en bloque el hilio hepático), ya que su uso prolongado genera un daño isquémico que se ha asociado con una mayor tasa de recurrencia de la enfermedad metastásica. Si bien es posible hacer esta maniobra con interrupciones, hasta durante 120 minutos y de manera segura, el preacondicionamiento isquémico permite hacerlo por un tiempo mayor, también de manera segura ${ }^{29}$.

Uno de los parámetros que claramente impacta el pronóstico oncológico de los pacientes operados, es la posibilidad de una adecuada resección de la lesión tumoral con márgenes microscópicos negativos para patología tumoral; de hecho, este es el único factor que ha logrado impactar la supervivencia, la tasa de recurrencia y el tiempo libre de enfermedad, cuando se ofrece un tratamiento quirúrgico ${ }^{30}$.

Existen dos tipos de resección, la anatómica y la preservadora de parénquima. En varios estudios se ha demostrado que el tipo de resección no influye en forma importante en el pronóstico del paciente ${ }^{31-33}$. No obstante, las que preservan el parénquima se asocian con menores tasas de sangrado y de complicaciones que las resecciones anatómicas ${ }^{34}$; además, la preservación de más parénquima sano es esencial para la función hepática posoperatoria ${ }^{35,36}$.

La discusión se ha centrado en el tamaño necesario del margen de resección, es decir, la distancia entre la línea de resección y el borde microscópico de la lesión tumoral, por lo cual se han llevado a cabo múltiples estudios, todos con resultados variables. Are, et al. ${ }^{37}$, analizaron el impacto del tamaño del margen de resección en el pronóstico a largo plazo de estos pacientes; en total, se incluyeron 1.019 casos en los cuales se comparó la supervivencia a cinco años, según el tamaño del margen de resección. La supervivencia fue del $29 \%$ 
con márgenes menores de $1 \mathrm{~mm}$, de $35 \%$ con márgenes entre 1 y $5 \mathrm{~mm}$, de $40 \%$ con márgenes de 5 a $10 \mathrm{~mm}$, y de $45 \%$ con márgenes de más de $10 \mathrm{~mm}$. Se concluyó que, idealmente, los márgenes de resección en estas circunstancias deben ser de más de un $\mathrm{cm}$, pero que la imposibilidad para lograrlos no es una contraindicación para no resecar la lesión tumoral.

Por otro lado, Pawlik, et al. ${ }^{38}$, midieron la supervivencia a cinco años de los pacientes sometidos a cirugía y su relación con los márgenes de resección. Con márgenes microscópicamente positivos para tumor, la supervivencia fue de $17,1 \% \mathrm{y}$, con márgenes negativos, fue de $63,8 \%$; no hubo variación importante según el tamaño del margen de resección: $62,3 \%$ con márgenes de 1 a $4 \mathrm{~mm}, 71 \%$ con márgenes de 5 a $9 \mathrm{~mm}$ y $63 \%$ en márgenes de más de $1 \mathrm{~cm}$.

Por lo anterior, y teniendo en cuenta que el promedio de la diseminación tumoral microscópica es de $4 \mathrm{~mm}$ con respecto al margen macroscópico, se recomienda practicar la resección dejando márgenes de un $\mathrm{cm}$ de ancho, para garantizar la ausencia de enfermedad tumoral residual.

\section{Cirugía mínimamente invasiva}

Con el avance de las técnicas mínimamente invasivas, ahora es posible realizar procedimientos mayores completamente laparoscópicos de manera segura, con resultados oncológicos equiparables a los de la cirugía abierta y con una aceptable morbimortalidad ${ }^{39,40}, \mathrm{y}$, además, con menor tasa de sangrado, menor tasa de infección y una recuperación más rápida ${ }^{41-45}$. Para conseguir estos resultados, Vigano, et al., consideran necesaria una adecuada curva de entrenamiento y practicar, como mínimo, 60 procedimientos, lo cual solo es posible en centros de alta complejidad y con gran volumen de casos de este tipo ${ }^{46}$.

\section{Ecografía intraoperatoria}

Desde su descripción por Makuuchi en 1977, el examen hepático intraoperatorio mediante ecografía ha venido ganando popularidad, hasta convertirse en un componente fundamental de la cirugía hepática moderna. Este método diagnóstico es esencial para practicar una resección preservadora de parénquima, ya que permite minimizar los márgenes de resección mientras se conserva la radicalidad oncológica, disminuyendo la resección innecesaria de tejido ${ }^{47,48}$.
La ecografía intraoperatoria permite confirmar las metástasis ya conocidas e identificar aquellas que pasaron inadvertidas en los estudios previos de extensión, particularmente las de menos de un $\mathrm{cm}$, en las cuales es menor el rendimiento diagnóstico de la resonancia magnética y de la tomografía axial. Además, hace posible observar las relaciones vasculares y biliares de las lesiones neoplásicas, e identificar el flujo portal, como guía para el tipo de resección o la ablación necesaria, y está disponible también por vía laparoscópica.

En una revisión de Ruzzenente, et al., la ecografía intraoperatoria con contraste permitió identificar lesiones adicionales en $14 \%$ de los casos y se cambió la conducta quirúrgica en el $18 \%$, lo cual mejoró la tasa de resección con márgenes negativos para tumor $\left(\mathrm{R}_{0}\right)^{49}$.

\section{Técnicas ablativas}

La ablación consiste en destruir el tumor hepático sin resecarlo. Los procedimientos ablativos se dividen en dos grandes grupos, la ablación regional transarterial y la ablación local ${ }^{50}$. En la ablación regional transarterial, se hacen llegar agentes quimioterápicos al tumor por medio de su irrigación, para inducir isquemia citotóxica. En la ablación local, se induce necrosis tumoral mediante la inyección de agentes citotóxicos o sustancias químicas que producen isquemia, o mediante la transmisión de energía térmica directamente al tejido tumoral ${ }^{51}$.

Se han descrito múltiples técnicas para el manejo ablativo de las metástasis hepáticas, y la más frecuentemente utilizada es la ablación por radiofrecuencia. En varios estudios se han demostrado mejores resultados oncológicos al comparar la ablación con el manejo paliativo; sin embargo, cuando se compara con la resección quirúrgica, muestra una menor supervivencia, ya que la ausencia de una necrosis completa de coagulación y la persistencia de células tumorales residuales después del procedimiento llevan a que las tasas de recurrencia sean mayores ${ }^{52}$. Esto es particularmente cierto en lesiones mayores de $2,5 \mathrm{~cm}$.

Por lo tanto, todos los pacientes con lesiones susceptibles de manejo quirúrgico deben ser sometidos a cirugía, reservando las terapias ablativas únicamente para aquellos que por sus condiciones clínicas no pueden ser operados, o para aquellos pacientes cuyas lesiones se localicen profundamente en el parénquima hepático 
y sean de tamaño pequeño, requiriendo procedimientos quirúrgicos complejos para su resección con pérdida de gran cantidad de parénquima hepático sano; en este último grupo de pacientes, se puede acompañar con la resección quirúrgica de otras lesiones a las cuales, por su localización, se puede acceder más fácilmente ${ }^{53}$.

\section{Volumen hepático funcional residual}

El volumen funcional residual del hígado se refiere al volumen de parénquima hepático que queda después de una resección quirúrgica, ya sea por enfermedad benigna o maligna. Su entendimiento es esencial, ya que, si bien el ser humano es incapaz de vivir sin hígado, puede tolerar resecciones hepáticas amplias siempre que se conserve un volumen de parénquima que cumpla las funciones orgánicas, inmunológicas, metabólicas y digestivas normales.

Está claro que un volumen hepático funcional residual insuficiente lleva a disfunción hepática posoperatoria, que provocará la muerte del paciente. Así las cosas, garantizar un adecuado volumen funcional residual es importante durante la planeación de un procedimiento quirúrgico que involucre la resección hepática en cualquier proporción. Su cálculo se hace mediante una volumetría hepática por resonancia magnética o tomografía axial, las cuales permiten la reconstrucción tridimensional del hígado residual futuro y su comparación con el volumen total hepático, lo cual da como resultado un porcentaje que será la guía para definir la posibilidad o imposibilidad de practicar la resección quirúrgica (figura 3 ).
En general, se acepta como guía para la práctica de cualquier procedimiento quirúrgico que involucre la resección hepática, la regla 20/30/40. Según esta regla: el volumen funcional residual necesario cuando el parénquima hepático está sano, debe ser de, por lo menos, el $20 \%$; cuando el parénquima está sano pero va a someterse a quimioterapia, se requiere un remanente hepático de más del $30 \%{ }^{54}$, y cuando el hígado es cirrótico, se requiere un volumen funcional residual de más del $40 \%{ }^{55-57}$. Esto garantiza intervenciones quirúrgicas seguras con poca morbimortalidad posoperatoria. Como todos estos pacientes se encuentran con enfermedad diseminada que requerirá el uso de quimioterapia, se debe garantizar, por lo menos, un volumen funcional residual de más del $30 \%$ con el fin de evitar la disfunción hepática posoperatoria.

\section{Métodos de hipertrofia hepática}

Las resecciones oncológicas hepáticas se ven condicionadas por la preservación de un adecuado remanente funcional y la posibilidad de resecar las lesiones tumorales. Tradicionalmente, cuando las lesiones metastásicas podían ser resecadas pero el remanente hepático era insuficiente, los pacientes recibían solamente tratamiento paliativo. Recientemente, se ha aprovechado la gran capacidad hepática de proliferar bajo determinados estímulos, para aumentar el volumen funcional residual y poder ofrecer una resección quirúrgica con fines curativos.

El principal estímulo de la hipertrofia hepática es la disrupción del flujo portal ${ }^{58}$; esta, al inducir isquemia en

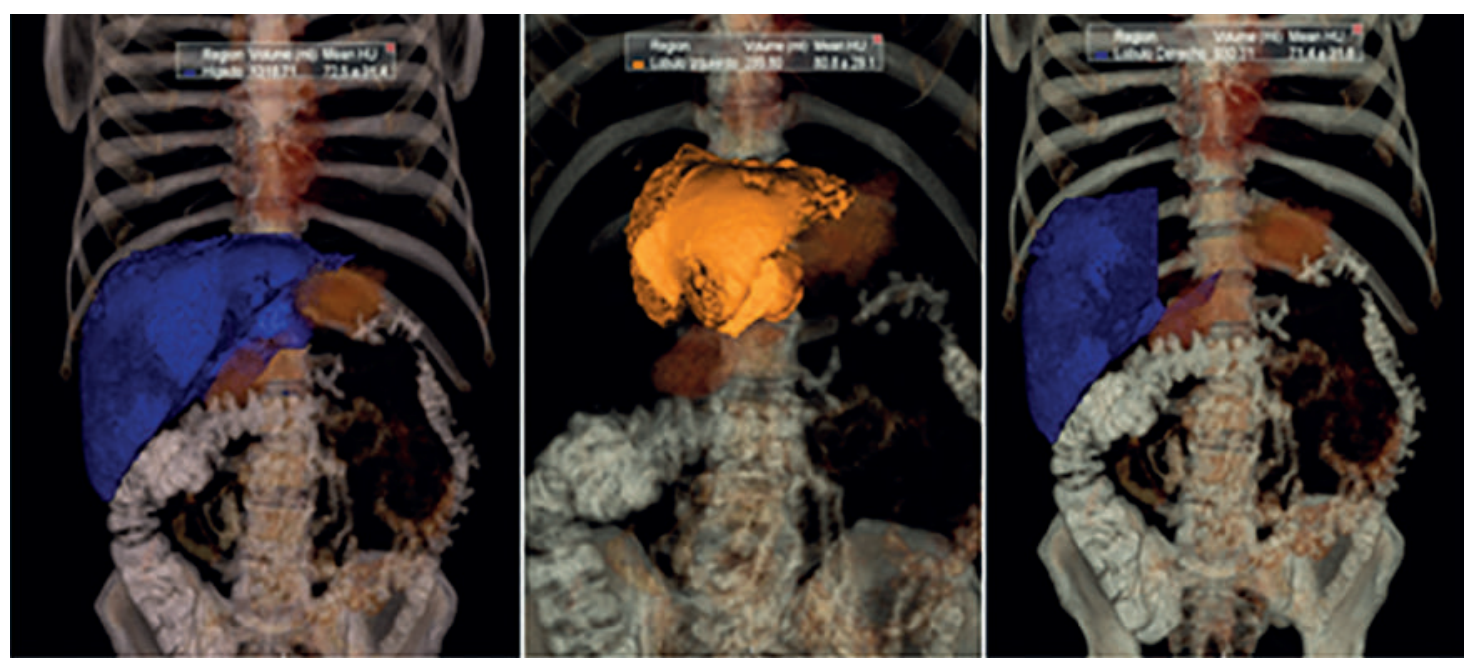

FIgURA 3. Volumetría hepática para el cálculo del volumen funcional residual 
el segmento comprometido, genera estímulos mediados por hormonas y factores de crecimiento ${ }^{59-61}$ que inducen hipertrofia del parénquima hepático del segmento no comprometido, aumentando así el volumen funcional residual y haciendo posible la resección quirúrgica. Sin embargo, estos mismos estímulos que inducen la hipertrofia del parénquima hepático sano, inducen también el crecimiento de las células malignas, lo cual propicia la progresión de los tumores y su conversión en irresecables. Por esta razón, en este tipo de situaciones se recomienda hacer un seguimiento estrecho del volumen hepático y de las metástasis, y adicionar esquemas de quimioterapia para evitar la progresión maligna.

A pesar del uso de estas técnicas, la progresión de la lesión tumoral sigue siendo la principal causa de imposibilidad de practicar la resección, con una tasa global del $10 \%$, seguida de la inadecuada hipertrofia del parénquima hepático que se presenta en el $2 \%$ de los casos en la actualidad ${ }^{62}$. En la mayoría de los casos, esta se explica por la circulación intraparenquimatosa entre ambos lóbulos, la cual permite la irrigación colateral del segmento isquémico $y$, por ende, la inadecuada hipertrofia del que se pretende conservar ${ }^{63,64}$.

La embolia o la ligadura portal se pueden practicar mediante cirugía abierta o por vía percutánea y facilitan la hipertrofia suficiente para proceder a la cirugía en $85 \%$ de los casos ${ }^{65,66}$. La hipertrofia calculada del remanente hepático es de $12 \%$ del volumen total hepático o de $37 \%$ del volumen funcional residual en cuatro a ocho semanas ${ }^{67,68}$. La partición hepática que consiste en individualizar quirúrgicamente ambos lóbulos hepáticos, permite separar el flujo portal de cada uno de ellos y evita la circulación intraparenquimatosa, lo cual aumenta la tasa de hipertrofia hepática deseada de manera más efectiva $^{69,70}$.

La hepatectomía por etapas, descrita por Adam en el $2000^{71}$, es una modalidad quirúrgica recomendada para tumores irresecables con metástasis hepáticas bilobares. En la primera cirugía, se reseca la mayor cantidad posible de metástasis, evitando la disfunción hepática posoperatoria. La segunda hepatectomía se decide con base en la regeneración hepática y la posibilidad de resecar las lesiones restantes, habiendo hecho control tumoral con quimioterapia entre ambas cirugías ${ }^{72}$. La combinación de embolia portal con hepatectomía por etapas ha demostrado buenos resultados. En la primera cirugía, se llevan a cabo la embolia portal del segmento más comprometido y la resección de las metástasis del futuro remanente hepático para evitar la progresión tumoral $\mathrm{y}$, en un segundo tiempo quirúrgico, cuatro a seis semanas después, la resección de las lesiones restantes.

Recientemente, se ha combinado la embolia portal con la partición hepática en el contexto de una hepatectomía por etapas, alcanzándose una importante hipertrofia del remanente hepático, de 61 a $93 \%$, en 9 a 14 días ${ }^{73-76}$. Esto permite ofrecer una cirugía temprana con fines curativos en 86 a $100 \%$ de los casos ${ }^{77-82}$. La principal desventaja de este método es la gran morbilidad que se presenta y, por ende, la selección de los pacientes debe ser cuidadosa para garantizar buenos resultados ${ }^{83,84}$.

\section{Tratamiento de las metástasis sincrónicas}

El manejo de las metástasis sincrónicas en pacientes con cáncer colorrectal representa un reto quirúrgico, no solo porque biológicamente son más agresivas ${ }^{85} \mathrm{y}$ tienen un peor pronóstico oncológico, sino, también, por la presencia de dos lesiones tumorales simultáneas que deben ser resecadas lo más pronto posible.

Se han propuesto numerosos abordajes para el tratamiento en estos casos; sin embargo, no hay consenso claro sobre cuál es el mejor método y cuál es el abordaje ideal, por lo que se analizan cada uno de los esquemas terapéuticos propuestos, con sus ventajas y desventajas, y posteriormente, se propone uno basado en los resultados obtenidos hasta la fecha ${ }^{86}$.

El abordaje tradicional o clásico consiste en la resección quirúrgica del tumor primario en un primer tiempo quirúrgico $\mathrm{y}$, posteriormente, quimioterapia, radioterapia o ambas, para proceder a la resección de las lesiones hepáticas en un segundo tiempo quirúrgico, tres a seis meses después. El tumor primario se debe resecar de manera temprana, considerando que es el foco de las metástasis y los síntomas del paciente, para evitar la progresión de la enfermedad.

La principal desventaja de esta secuencia terapéutica es que permite la progresión de las metástasis hepáticas, las cuales pueden tornarse irresecables, particularmente cuando se presentan complicaciones en el primer 
acto quirúrgico que impidan el inicio temprano de la quimioterapia. Según un estudio de 2012 basado en la Liver Met Survey, que comprendió un periodo de 10 años, se encontró que menos del $30 \%$ de los pacientes sometidos a este esquema, lograban ser sometidos a la resección quirúrgica de las metástasis ${ }^{87}$, por lo cual, en la actualidad, este esquema se reserva solo para los pacientes en los cuales el tumor primario se encuentra sintomático o presenta alguna complicación.

El abordaje simultáneo consiste en la resección de la lesión tumoral primaria y de las metástasis hepáticas en un mismo acto quirúrgico, seguida de quimioterapia, radioterapia o ambas. Este permite la resección temprana de ambas lesiones, impidiendo el retraso quirúrgico, particularmente de la lesión metastásica ${ }^{88}$, y permitiendo el inicio temprano de la quimioterapia para mejorar el pronóstico oncológico.

Su principal desventaja es el aumento de la morbimortalidad posoperatoria al someter al paciente a dos procedimientos quirúrgicos en el mismo acto ${ }^{89,90}$. Recientemente, en varios estudios se ha demostrado que las complicaciones posoperatorias son equiparables a las de otros esquemas cuando uno de los dos procedimientos, o ambos, se consideran menores (tabla 2) ${ }^{91,92}$; lo contrario ocurre cuando ambas cirugías se consideran mayores, y la morbimortalidad sí se aumenta de forma significativa.

Por lo tanto, actualmente se recomienda que este esquema terapéutico se utilice cuando ambas cirugías se consideran menores, o al menos una de ellas, y siempre en pacientes cuidadosamente seleccionados que puedan soportar adecuadamente la resección simultánea de ambas lesiones (tabla 2).

La estrategia inversa propuesta por Mentha, et al., en el 2008, consiste en administrar inicialmente tres a seis ciclos de quimioterapia sistémica, seguidos por la resección de las metástasis hepáticas y, posteriormente, en un segundo tiempo quirúrgico, resecar el tumor primario, todo esto con quimioterapia durante el intervalo entre ambas cirugías ${ }^{93}$. Su postulación se basa en el hecho de que el pronóstico está determinado por la posibilidad de resecar las metástasis, y no por la progresión del tumor primario o sus complicaciones potenciales.

Las ventajas de este esquema son que, al iniciarse con la quimioterapia, hay un beneficio sistémico que se
TABLA 2.

Clasificación de las cirugías hepáticas y colorrectales como menores o mayores

\begin{tabular}{lll}
\hline Órgano & \multicolumn{1}{c}{ Cirugía mayor } & Cirugía menor \\
\hline Colon & $\begin{array}{l}\text { Recto } \\
\text { Colon izquierdo }\end{array}$ & Colon derecho \\
\hline Hígado & $\begin{array}{l}\text { Hepatectomía de más } \\
\text { de tres segmentos }\end{array}$ & $\begin{array}{l}\text { Hepatectomía } \\
\text { de menos de tres } \\
\text { segmentos }\end{array}$ \\
\hline
\end{tabular}

garantiza de manera temprana y, además, las metástasis hepáticas se resecan rápidamente disminuyendo así el riesgo de que se conviertan en irresecables; de hecho, muchas de ellas disminuyen de tamaño gracias a la quimioterapia, lo cual permite resecciones menores dejando más parénquima sano funcional.

La principal desventaja de este método es que podría permitir la progresión tumoral primaria y sus subsecuentes complicaciones. Sin embargo, por un lado, se ha demostrado que, cuando se administra quimioterapia neoadyuvante, se disminuye el tamaño de la lesión primaria y se evita su progresión ${ }^{94}, \mathrm{y}$, por el otro, cuando el tumor primario es asintomático y se inicia este esquema, más del $90 \%$ de los pacientes no desarrollan complicaciones asociadas con la lesión primaria. De igual forma, de 66 a $81 \%$ de los pacientes con este esquema terapéutico lo terminan satisfactoriamente, lográndose la resección completa del tumor y un impacto en la supervivencia.

Por esta razón, la estrategia inversa se considera el tratamiento de elección en pacientes con cáncer de colon o recto con metástasis hepáticas, en el que el tumor primario es asintomático y las lesiones hepáticas son resecables, limítrofes o, incluso, irresecables ${ }^{95}$.

En 2008, Grundmann, et al., propusieron una modificación al esquema de Mentha, ya que consideraron que los pacientes con lesiones hepáticas resecables desde un inicio no se benefician de la quimioterapia neoadyuvante ${ }^{96}$, pues de 7 a $37 \%$ no tienen una reacción terapéutica satisfactoria y las metástasis progresan hasta el punto de volverse irresecables, lo cual oscurece el pronóstico oncológico. En otros, por el contrario, desaparecen las lesiones (2-36\%) ${ }^{97}$, convirtiéndose en un reto quirúrgico para el cirujano, ya que se ha demostrado que en $80 \%$ de los casos en los cuales hay resolución macroscópica de la lesión, persiste la actividad tumoral 
microscópica, lo cual lleva a la resección incompleta de las lesiones tumorales ${ }^{98}$; esto aumenta las tasas de recurrencia y disminuye la supervivencia de los pacientes. De esta manera, se considera que los pacientes con metástasis que pueden ser sometidos inmediatamente a cirugía, deben manejarse con este esquema para evitar las anteriores situaciones.

A pesar de las diferentes estrategias propuestas, no existe claridad sobre cuándo se debe usar cada uno de los métodos y si en algún caso en particular es preferible el uno sobre el otro. Sin embargo, en algunos estudios comparativos se ha demostrado una supervivencia equivalente con los diferentes esquemas terapéuticos, siempre y cuando se logren implementar totalmente ${ }^{99,100}$.

Por lo tanto, a la hora de seleccionar el mejor esquema para cada paciente, el factor fundamental es que corresponda a sus características clínicas y que sea posible llevarlo a cabo por completo. De esta forma, la única indicación para un abordaje tradicional sería la presencia de un tumor primario sintomático o complicado, ya que la tasa de éxito con este esquema es de solo el $30 \%$, mientras que, con la estrategia inversa es de $80 \%$. Cuando el tumor primario es asintomático y el paciente se encuentra en condiciones clínicas adecuadas para practicar simultáneamente los procedimientos, esta sería la mejor opción, siempre que ambos procedimientos no sean considerados como mayores; si este es el caso, se recomienda un abordaje retrógrado modificado, si la lesión es resecable desde un inicio, o uno retrógrado, si es limítrofe. En la figura 4 se resume el algoritmo de manejo propuesto.

\section{Tratamiento de las metástasis metacrónicas}

El abordaje quirúrgico de las metástasis metacrónicas es menos polémico que el de las sincrónicas, ya que biológicamente son menos agresivas y solo hay un órgano comprometido con las lesiones tumorales. Por lo

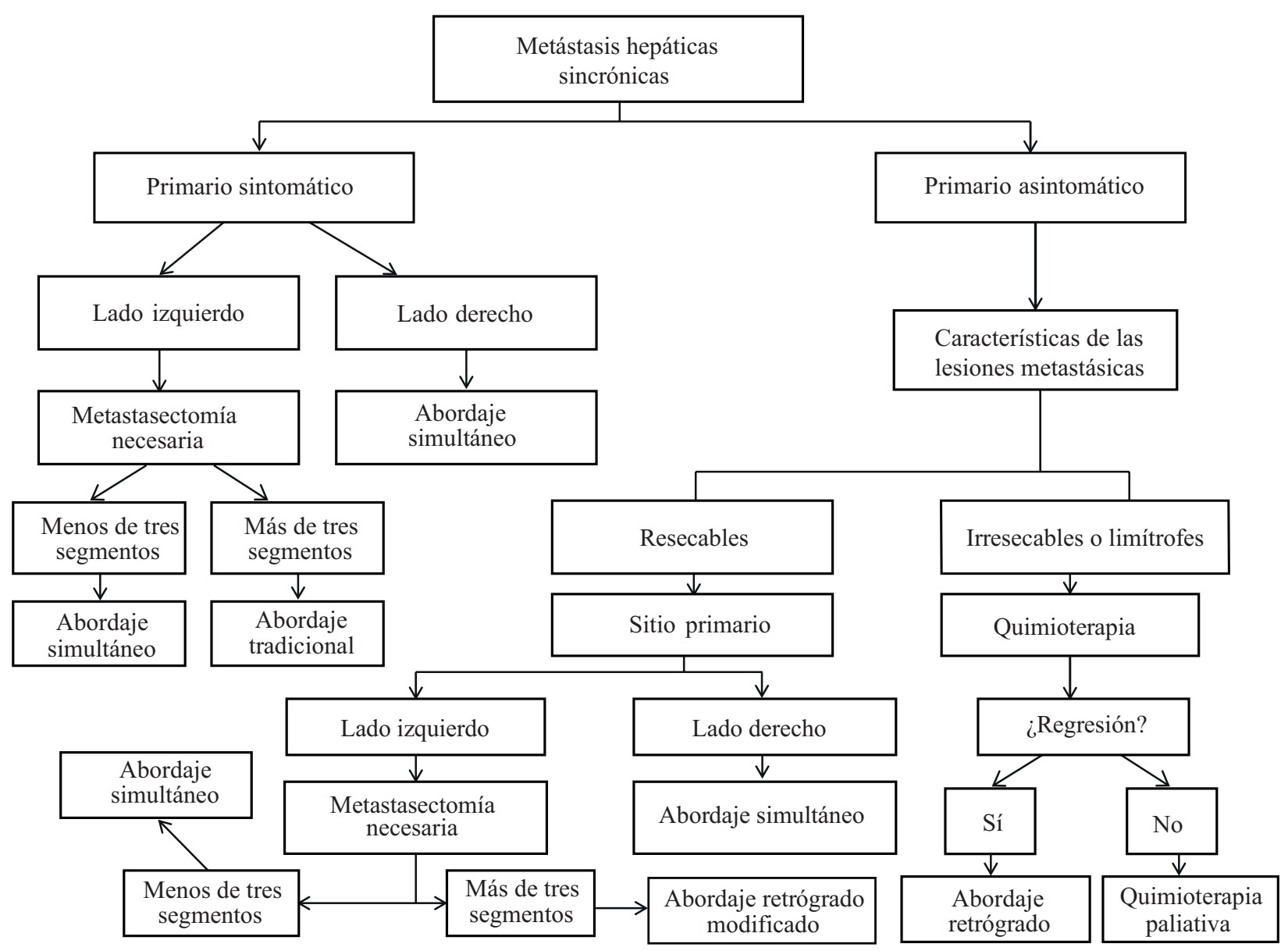

FIgURA 4. Tratamiento de las metástasis hepáticas sincrónicas en cáncer colorrectal 
anterior, el momento de la resección quirúrgica se verá determinado únicamente por el tamaño de las lesiones al momento del diagnóstico.

Las metástasis pequeñas que pueden ser resecadas de entrada se benefician de cirugía de manera temprana $y$, posteriormente, quimioterapia con el fin de evitar la progresión tumoral durante la neoadyuvancia.

Para las lesiones grandes con posibilidad limítrofe de ser resecadas, se debe administrar quimioterapia neoadyuvante, con el fin de disminuir su tamaño y permitir una adecuada resección quirúrgica, preservando la mayor cantidad de parénquima hepático sano posible.

Cuando el volumen residual funcional sea insuficiente, se deben usar técnicas de hipertrofia hepática y, cuando las lesiones sean múltiples y bilobares, la hepatectomía por etapas puede ser de utilidad con el fin de incrementar la posibilidad de resección.

\section{Metástasis identificadas durante la cirugía}

Una de las situaciones más frecuentes y a la vez más difíciles de manejar para el cirujano es encontrar metástasis hepáticas durante la resección de un tumor primario de colon o recto. Esto puede suceder cuando el tumor primario se inicia con obstrucción, perforación o ambas, haciendo urgente la cirugía, sin estudios previos de extensión, o cuando las metástasis no se detectan con los estudios de imágenes.

Esto último es cada vez menos frecuente, ya que la sensibilidad y la especificidad de la tomografía axial y la resonancia magnética son cada vez mayores para detectar metástasis cada vez más pequeñas; sin embargo, es bien sabido que su rendimiento diagnóstico disminuye cuando las lesiones son de menos de un $\mathrm{cm}$ y se pueden pasar por alto metástasis hepáticas ${ }^{101-103}$.

Cuando el tiempo que transcurre entre las imágenes y la cirugía es prolongado, se corre el riesgo de que se presente progresión tumoral e, incluso, aparezcan nuevas metástasis que no se identificaban en los estudios imaginológicos; por esta razón, se recomienda que no deben pasar más de cuatro semanas entre el estudio imaginológico y el procedimiento quirúrgico.

Un factor determinante para decidir la conducta en este tipo de situaciones, es la disponibilidad de un cirujano hepatobiliar que pueda practicar el procedimiento hepático en ese momento. En caso de no estar disponible este especialista, se recomienda resecar la lesión primaria, independientemente de los demás factores, ya que el paciente se encuentra anestesiado y preparado para dicho procedimiento quirúrgico. Cuando el cirujano hepatobiliar esté disponible, se debe determinar si el tumor primario es sintomático o asintomático y establecer el tamaño de las metástasis hepáticas sincrónicas (figura 5).

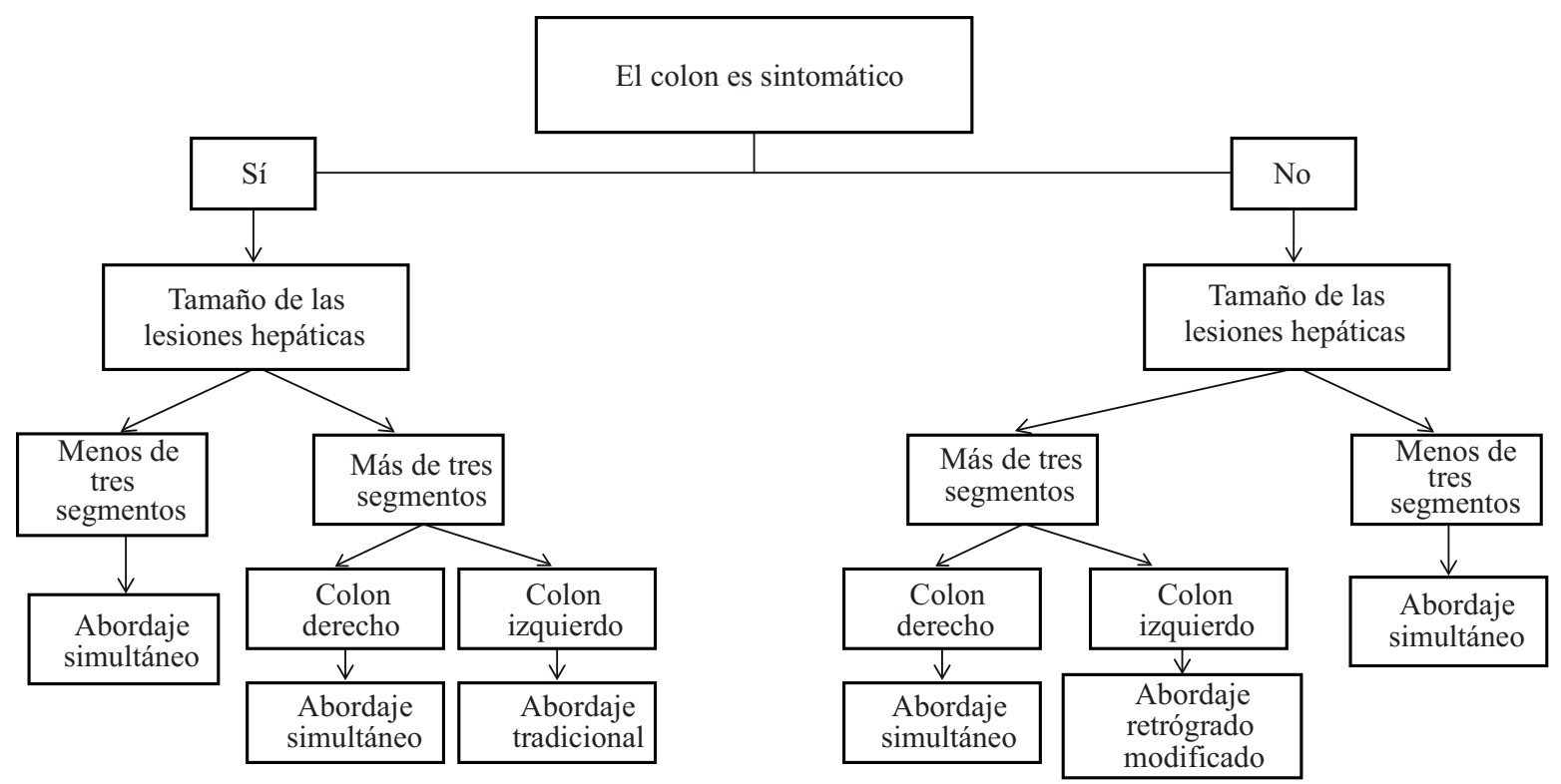

FIGURA 5. Tratamiento de las metástasis hepáticas sincrónicas no conocidas previamente y diagnosticadas durante la cirugía 


\section{Conclusiones}

Las metástasis hepáticas en el cáncer colorrectal son frecuentes y, tradicionalmente, se consideraban irresecables, por lo cual solamente se ofrecía tratamiento paliativo. Actualmente, las alternativas terapéuticas han aumentado a tal punto, que se puede optar por procedimientos curativos con impacto significativo en la supervivencia, todos los cuales involucran la resección quirúrgica de las lesiones y la quimioterapia sistémica.
El entendimiento a fondo de la enfermedad y el conocimiento de los esquemas terapéuticos disponibles, tanto en caso de metástasis sincrónicas como metacrónicas, permiten ofrecerles al paciente y a su familia alternativas de tratamiento que permiten, no solo aumentar la supervivencia de la enfermedad, sino también, una mejor calidad de vida.

\title{
Hepatic metástases in colorectal cancer: therapeutic strategies and current recommendations
}

\begin{abstract}
Metastatic colorectal cancer in the liver is a frequent pathology that until a few years ago was considered only for palliative management. However, with the recent advent of new techniques and advances to improve the resectability of such lesions and the new schemes of chemotherapy, oncological resection of these lesions can be performed, extracting all macroscopic tumor and achieving a positive impact on patient's survival.

Multiple schemes have been postulated for the management of synchronous lesions in order to improve patient survival, although there is no consensus about which is the best scheme in these cases; it seems that the most important factor is to complete successfully the proposed scheme. In general, the current trend is to perform the liver resection first unless the primary tumor is symptomatic, in which case a colectomy should be performed as the initial approach. Simultaneous resections are part of the therapeutic arsenal in these patients. Many groups suggest not to associate a low rectal resection with a major hepatectomy. On the other hand, metachronic lesions should be resected based on their size and the possibility of resection should be considered once the diagnosis has been made.

It is important to take into account that even if a complete resection of the tumor and R0 margins are achieved, recurrences are frequent because microscopic tumor dissemination is already present; therefore, the addition of chemotherapy regimens should be mandatory to Improve the oncological prognosis of these patients.
\end{abstract}

Key words: Neoplasms; liver; colonic neoplasms; neoplasm metastasis; neoplasms, second primary; hepatectomy; clinical protocols; survival.

\section{Referencias}

1. Siegel RL, Miller KD, Jemal A. Cancer Statistics, 2017. CA Cancer J Clin. 2017;67:7-30.

2. Ferlay J, Shin HR, Bray F, Forman D, Mathers C, Parkin DM. Estimates of worldwide burden of cancer in 2008: GLOBOCAN 2008. Int J Cancer. 2010;127:2893-917.

3. Siegel R, Naishadham D, Jemal A. Cancer statistics, 2012. CA Cancer J Clin. 2012;62:10-29.

4. Uribe M, Paqui L, Uribe-Echevarría S, Romanque P, Azabache V. Resección de metástasis hepáticas en cáncer de colon. Nuevas perspectivas. Gastroenterol Latinoam. 2014;25:50-3.

5. Manfredi S, Lepage C, Hatem C, Coatmeur O, Faivre J, Bouvier AM. Epidemiology and management of liver metastases from colorectal cancer. Ann Surg. 2006;244:254-9
6. McMillan DC, McArdle CS. Epidemiology of colorectal liver metastases. Surg Oncol. 2007;16:3-5.

7. Lochan R, White SA, Manas DM. Liver resection for colorectal liver metastasis. Surg Oncol. 2007;16:33-45.

8. Leporrier J, Maurel J, Chiche L, Bara S, Segol P, Launoy G. A population-based study of the incidence, management and prognosis of hepatic metastases from colorectal cancer. Br J Surg. 2006;93:465-74.

9. Tzeng CW, Aloia TA. Colorectal liver metastases. J Gastrointest Surg. 2013;17:195-202.

10. Adam R, Vinet E. Regional treatment of metastasis: Surgery of colorectal liver metastases. Ann Oncol. 2004;15(Suppl.4): 103-6. 
11. Simmonds PC, Primrose JN, Colquitt JL, Garden OJ, Poston GJ, Rees M. Surgical resection of hepatic metastases from colorectal cancer: A systematic review of published studies. Br J Cancer. 2006;94:982-99.

12. Ruiz-Figueroa EF, Celis-Zapata J, Berrospi-Espinoza F, PayetMeza E. Tratamiento quirúrgico de las metástasis hepáticas de cáncer colorectal. Rev Gastroenterol Perú. 2000;20:376-83.

13. Fontan R, Herman P, Pugliese V, Vinicius Perini M, Ferreira Coelho F, Cecconello I. Surgical outcomes and prognostic factors in patients with synchronous colorectal liver metastases. Arq Gastroenterol. 2014:51;4-9.

14. Yamada H, Kondo S, Okushiba S, Morikawa T, Katoh H. Analysis of predictive factors for recurrence after hepatectomy for colorectal liver metastases. World J Surg. 2001;25:1129-33.

15. De Jong MC, Pulitano C, Ribero D, Strub J, Mentha G, Schulick $\mathrm{RD}$, et al. Rates and patterns of recurrence following curative intent surgery for colorectal liver metastasis: An international multi-institutional analysis of 1,669 patients, Ann Surg. 2009;250:440-8.

16. Abdalla EK, Vauthey JN, Ellis LM, Pollock R, Broglio KR, Hess $\mathrm{K}$, et al. Recurrence and outcomes following hepatic resection, radiofrequency ablation, and combined resection/ablation for colorectal liver metastases. Ann Surg. 2004;239:818-25.

17. D'Angelica M, Kornprat P, Gonen M, DeMatteo RP, Fong Y, Blumgart LH, et al. Effect on outcome of recurrence patterns after hepatectomy for colorectal metastases. Ann Surg Oncol. 2011;18:1096-103.

18. Hashimoto M, Kobayashi T, Ishiyama T, Ide K, Ohira M, Tahara $\mathrm{H}$, et al. Efficacy of repeat hepatectomy for recurrence following curative hepatectomy for colorectal liver metastases: A retrospective cohort study of 128 patients. Int J Surg. 2016;36:96-103.

19. Nordlinger B, Guiguet M, Vaillant JC, Balladur P, Boudjema K, Bachellier $\mathrm{P}$, et al. Surgical resection of colorectal carcinoma metastases to the liver. A prognostic scoring system to improve case selection, based on 1,568 patients. Cancer. 1996;77:125462 .

20. Tsai MS, Su YH, Ho MC, Liang JT, Chen TP, Lai HS, et al. Clinico-pathological features and prognosis in resectable synchronous and metachronous colorectal liver metastasis. Ann Surg Oncol. 2007;14:786-94.

21. Nordlinger B, van Cutsem E, Rougier P, Köhne CH, Ychou M, Sobrero A, et al. European Colorectal Metastases Treatment Group: Does chemotherapy prior to liver resection increase the potential for cure in patients with metastatic colorectal cancer? A report from the European Colorectal Metastases Treatment Group. Eur J Cancer. 2007;43:2037-45.

22. Nabil I. Treatment of colorectal liver metastases. Ismaili World Journal of Surgical Oncology. 2011;9:154.

23. Simmonds PC, Primrose JN, Colquitt JL, Garden OJ, Poston GJ, Rees M. Surgical resection of hepatic metastases from colorectal cancer: A systematic review of published studies. Br J Cancer. 2006;94:982-99.
24. Adams RB, Aloia TA, Loyer E, Pawlik TM, Taouli B, Vauthey JN. Selection for hepatic resection of colorectal liver metastases: Expert consensus statement. HPB. 2013;15:91-103.

25. Grundmann RT. Current state of surgical treatment of liver metastases from colorectal cancer. World J Gastrointest Surg. 2011;3:183-96.

26. Akgül O, Çetinkaya E, Ersöz S, Tez M. Role of surgery in colorectal cancer liver metastases. World J Gastroenterol. 2014;20:6113-22.

27. Pawlik TM, Schulick RD, Choti MA. Expanding criteria for resectability of colorectal liver metastases. Oncologist. 2008;13:51-64.

28. Grundmann RT, Hermanek P, Merkel S, Germer CT, Grundmann RT, Hauss J, et al. Diagnosis and treatment of colorectal liver metastases - workflow. Zentralbl Chir. 2008;133:267-84.

29. Nijkamp MW, van der Bilt JD, Snoeren N, Hoogwater FJ, van Houdt WJ, Molenaar IQ, et al. Prolonged portal triad clamping during liver surgery for colorectal liver metastases is associated with decreased time to hepatic tumour recurrence. Eur J Surg Oncol. 2010;36:182-8.

30. Herman P, Pinheiro RS, Mello ES, Lai Q, Lupinacci RM, Perini MV, et al. Surgical margin size in hepatic resections for colorectal metastasis: Impact on recurrence and survival. Abcd Arq Bras Cir Dig. 2013;26:309-14.

31. Sarpel U, Bonavia AS, Grucela A, Roayaie S, Schwartz ME, Labow DM. Does anatomic versus non-anatomic resection affect recurrence and survival in patients undergoing surgery for colorectal liver metastasis? Ann Surg Oncol. 2009;16:379-84.

32. Zorzi D, Mullen JT, Abdalla EK, Pawlik TM, Andres A, Muratore A, et al. Comparison between hepatic wedge resection and anatomic resection for colorectal liver metastases. J Gastrointest Surg. 2006;10:86-94.

33. Stewart GD, O'Súilleabháin CB, Madhavan KK, Wigmore SJ, Parks RW, Garden OJ. The extent of resection influences outcome following hepatectomy for colorectal liver metastases. Eur J Surg Oncol. 2004;30:370-6.

34. Kostov DV, Kobakov GL. Segmental liver resection for colorectal metastases. J Gastrointestin Liver Dis. 2009;18:447-53.

35. Álvarez FA, Sánchez-Claria R, Oggero S, de Santibañes E. Parenchymal-sparing liver surgery in patients with colorectal carcinoma liver metastases. World J Gastrointest Surg. 2016;8:407-23.

36. Vinicius Perini M, Ferreira Coelho F, Ferrari Makdissi F, Miccelli Lupinacci R, Autran Machado M, Herman P. Estrategias para aumentar a ressecabilidade em pacientes com metástases hepáticas de tumores colorretais. Abcd Arq Bras Cir Dig. 2011;24:324-7.

37. Are C, Gonen M, Zazzali K, Dematteo RP, Jarnagin WR, Fong $\mathrm{Y}$, et al. The impact of margins on outcome after hepatic resection for colorectal metastasis. Ann Surg. 2007;246:295-300.

38. Pawlik TM, Scoggins CR, Zorzi D, Abdalla EK, Andres A, Eng $\mathrm{C}$, et al. Effect of surgical margin status on survival and site 
of recurrence after hepatic resection for colorectal metastases. Ann Surg. 2005;241:715-24.

39. Rostirolla R, Fontes P, Pinho M, Pedroso M, Masuko T, Novelli $\mathrm{P}$, et al. The impact of laparoscopic surgery in colorectal cancer resection with respect to the development of liver metastasis in the long-term. J Coloproctol (Rio J). 2016;36:8-15.

40. Micelli Lupinacci R, Autran Machado M, Arioni Lupinacci R, Herman P. Simultaneous left colectomy and standard hepatectomy performed by laparoscopy. Rev Col Bras Cir. 2011;38:139-41.

41. Simillis C, Constantinides VA, Tekkis PP, Darzi A, Lovegrove $\mathrm{R}$, Jiao L, et al. Laparoscopic versus open hepatic resections for benign and malignant neoplasms--a metaanalysis. Surgery. 2007;141:203-11.

42. Koffron AJ, Auffenberg G, Kung R, Abecassis M. Evaluation of 300 minimally invasive liver resections at a single institution: Less is more. Ann Surg. 2007;246:385-94.

43. Buell JF, Thomas MT, Rudich S, Marvin M, Nagubandi R, Ravindra KV, et al. Experience with more than 500 minimally invasive hepatic procedures. Ann Surg. 2008;248:475-86.

44. Castaing D, Vibert E, Ricca L, Azoulay D, Adam R, Gayet B. Oncologic results of laparoscopic versus open hepatectomy for colorectal liver metastases in two specialized centers. Ann Surg. 2009;250:849-55.

45. López-Ben S, Ranea A, Albiol MT, Falgueras L, Castro E, Casellas M, et al. Evolution of laparoscopic surgery in a high volume hepatobiliary unit: 150 consecutive pure laparoscopic hepatectomies. Cir Esp. 2017;95:261-7.

46. Vigano L, Laurent A, Tayar C, Tomatis M, Ponti A, Cherqui D. The learning curve in laparoscopic liver resection: Improved feasibility and reproducibility. Ann Surg. 2009;250:772-82.

47. Torzilli G, Montorsi M, Donadon M, Palmisano A, Del Fabbro $\mathrm{D}$, Gambetti A, et al. "Radical but conservative" is the main goal for ultrasonography-guided liver resection: Prospective validation of this approach. J Am Coll Surg. 2005;201:517-28.

48. Torzilli G, Montorsi M, Del Fabbro D, Palmisano A, Donadon M, Makuuchi M. Ultrasonographically guided surgical approach to liver tumours involving the hepatic veins close to the caval confluence. Br J Surg. 2006;93:1238-46.

49. Ruzzenente A, Conci S, Iacono C, Valdegamberi A, Campagnaro $\mathrm{T}$, Bertuzzo $\mathrm{F}$, et al. Usefulness of contrast-enhanced intraoperative ultrasonography (CE-IOUS) in patients with colorectal liver metastases after preoperative chemotherapy. J Gastrointest Surg. 2013;17:281-7.

50. Eisele RM. Advances in local ablation of malignant liver lesions. World J Gastroenterol. 2016;22:3885-91.

51. Boutros C, Somasundar P, Garrean S, Saied A, Espat NJ. Microwave coagulation therapy for hepatic tumors: Review of the literature and critical analysis. Surg Oncol. 2010;19:22-32.

52. Lu DS, Yu NC, Raman SS, Limanond P, Lassman C, Murray $\mathrm{K}$, et al. Radiofrequency ablation of hepatocellular carcinoma: Treatment success as defined by histologic examination of the explanted liver. Radiology. 2005;234:954-60.
53. Ito K, Govindarajan A, Ito H, Fong Y. Surgical treatment of hepatic colorectal metastasis: Evolving role in the setting of improving systemic therapies and ablative treatments in the 21st century. Cancer J. 2010;16:103-10.

54. Clavien PA, Petrowsky H, DeOlivera ML, Graf R. Strategies for safer liver surgery and partial liver transplantation. N Eng J Med. 2007;356:1545-59.

55. Garcea G, Ong SL, Maddern GJ. Predicting liver failure following major hepatectomy. Dig Liver Dis. 2009;41:798-806.

56. Tanaka K, Shimada H, Matsuo K, Ueda M, Endo I, Togo S. Remnant liver regeneration after two-stage hepatectomy for multiple bilobar colorectal metastases. Eur J Surg Oncol. 2007;33:329-35.

57. Tucker ON, Heaton N. The "small for size" liver syndrome. Curr Opin Crit Care. 2005;11:150-5.

58. Mortensen KE. Increased sinusoidal flow is not the primary stimulus to liver regeneration. Comp Hepatol. 2010:9:2.

59. Mortensen KE, Revhaug A. Liver regeneration in surgical animal models: A historical perspective and clinical implications. Eur Surg Res. 2011;46:1-18.

60. Michalopoulos GK, De Frances MC. Liver regeneration. Science. 1997;276:60-6.

61. Schlegel A, Lesurtel M, Melloul E, Limani P, Tschuor C, Graf $\mathrm{R}$, et al. ALPPS: From human to mice highlighting accelerated and novel mechanisms of liver regeneration. Ann Surg. 2014;260:839-47.

62. Abulkhir A, Limongelli P, Healey AJ, Damrah O, Tait P, Jackson $\mathrm{J}$, et al. Preoperative portal vein embolization for major liver resection: A meta-analysis. Ann Surg. 2008;247:49-57.

63. Riehle KJ, Dan YY, Campbell JS, Fausto N. New concepts in liver regeneration. J Gastroenterol Hepatol. 2011;26:203-12.

64. Wilms C, Mueller L, Lenk C, Wittkugel O, Helmke K, KrupskiBerdien $\mathrm{G}$, et al. Comparative study of portal vein embolization versus portal vein ligation for induction of hypertrophy of the future liver remnant using a mini-pig model. Ann Surg. 2008;247:825-34.

65. Capussotti L, Muratore A, Baracchi F, Lelong B, Ferrero A, Regge $\mathrm{D}$, et al. Portal vein ligation as an efficient method of increasing the future liver remnant volume in the surgical treatment of colorectal metastases. Arch Surg. 2008;143: 978-82.

66. Hemming AW, Reed AI, Howard RJ, Fujita S, Hochwald SN, Caridi JG, et al. Preoperative portal vein embolization for extended hepatectomy. Ann Surg. 2003;237:686-93.

67. Abdalla EK, Hicks ME, Vauthey JN. Portal vein embolization: Rationale, technique and future prospects. Br J Surg. 2001;88:165-75.

68. Pamecha V, Glantzounis G, Davies N, Fusai G, Sharma D, Davidson B. Long-term survival and disease recurrence following portal vein embolisation prior to major hepatectomy for colorectal metastases. Ann Surg Oncol. 2009;16:1202-7. 
69. Gall TM, Sodergren MH, Frampton AE, Fan R, Spalding DR, Habib NA, et al. Radio-frequency-assisted liver partition with portal vein ligation (RALPP) for liver regeneration. Ann Surg. 2015;261:e45-6.

70. Tanaka K, Endo I. ALPPS: Short-term outcome and functional changes in the future liver remnant. Ann Surg. 2015;262:e88-9.

71. Adam R, Laurent A, Azoulay D, Castaing D, Bismuth H. Twostage hepatectomy: A planned strategy to treat irresectable liver tumors. Ann Surg. 2000;232:777-85.

72. Jaeck D, Oussoultzoglou E, Rosso E, Greget M, Weber JC, Bachellier PA. Two-stage hepatectomy procedure combined with portal vein embolization to achieve curative resection for initially unresectable multiple and bilobar colorectal liver metastases. Ann Surg. 2004;240:1037-51.

73. Schnitzbauer AA, Lang SA, Goessmann H, Nadalin S, Baumgart J, Farkas SA, et al. Right portal vein ligation combined with in situ splitting induces rapid left lateral liver lobe hypertrophy enabling 2-staged extended right hepatic resection in small-forsize settings. Ann Surg. 2012;255:405-14.

74. Schadde E, Ardiles V, Slankamenac K, Tschuor C, Sergeant G, Amacker $\mathrm{N}$, et al. ALPPS offers a better chance of complete resection in patients with primarily unresectable liver tumors compared with conventional-staged hepatectomies: Results of a multicenter analysis. World J Surg. 2014;38:1510-9.

75. Sala S, Ardiles V, Ulla M, Alvarez F, Pekolj J, de Santibañes E. Our initial experience with ALPPS technique: Encouraging results. Updat Surg. 2012;64:167-72.

76. Hernández-Alejandro R, Bertens KA, Pineda-Solís K, Croome KP. Can we improve the morbidity and mortality associated with the associating liver partition with portal vein ligation for staged hepatectomy (ALPPS) procedure in the management of colorectal liver metastases? Surgery. 2015;157:194-201.

77. Nadalin S, Capobianco I, Li J, Girotti P, Konigsrainer I, Konigsrainer A. Indications and limits for associating liver partition and portal vein ligation for staged hepatectomy (ALPPS), lessons learned from 15 cases at a single centre. Z Gastroenterol. 2014;52:35-42.

78. Schadde E, Ardiles V, Robles-Campos R, Malago M, Machado R, Hernández-Alejandro R, et al. Early survival and safety of ALPPS: First report of the international ALPPS registry. Ann Surg. 2014;260:829-38.

79. Álvarez FA, Ardiles V, Sánchez-Claria R, Pekolj J, de Santibañes E. Associating liver partition and portal vein ligation for staged hepatectomy (ALPPS): Tips and tricks. J Gastrointest Surg. 2013;17:814-21.

80. Li J, Girotti P, Konigsrainer I, Ladurner R, Konigsrainer A, Nadalin S. ALPPS in right trisectionectomy: A safe procedure to avoid postoperative liver failure? J Gastrointest Surg. 2013;17:956-61.

81. Knoefel WT, Gabor I, Rehders A, Alexander A, Krausch M, Schulte J, et al. In situ liver transection with portal vein ligation for rapid growth of the future liver remnant in two-stage liver resection. Br J Surg. 2013;100:388-94.
82. Oldhafer KJ, Donati M, Jenner RM, Stang A, Stavrou GA. ALPPS for patients with colorectal liver metastases: Effective liver hypertrophy, but early tumor recurrence. World J Surg. 2014;38:1504-9.

83. Bertens KA, Hawel J, Lung K, Buac S, Pineda-Solis K, Hernández-Alejandro R. ALPPS: Challenging the concept of unresectability. A systematic review. Int J Surg. 2015;13:280-7.

84. Martins Torres OJ, Assunção Moraes-Junior JM, Lima E Lima $\mathrm{NC}$, Moraes AM. Ligadura da veia porta associada á transecção para hepatectomia em dois estágios (Alpps): uma nova abordagem nas ressecções hepáticas. Abcd Arq Bras Cir Dig. $2012 ; 254: 290-2$

85. Tsai MS, Su YH, Ho MC, Liang JT, Chen TP, Lai HS, et al. Clinico-pathological features and prognosis in resectable synchronous and metachronous colorectal liver metastasis. Ann Surg Oncol. 2007;14:786-94.

86. Ihnát P, Vávra P, Zonča P. Treatment strategies for colorectal carcinoma with synchronous liver metastases: Which way to go? World J Gastroenterol. 2015;21:7014-21.

87. Andres A, Toso C, Adam R, Barroso E, Hubert C, Capussotti L, et al. A survival analysis of the liver-first reversed management of advanced simultaneous colorectal liver metastases: A liver Met survey-based study. Ann Surg. 2012;256:772-9.

88. Brouquet A, Mortenson MM, Vauthey JN, Rodriguez-Bigas MA, Overman MJ, Chang GJ, et al. Surgical strategies for synchronous colorectal liver metastases in 156 consecutive patients: Classic, combined or reverse strategy? J Am Coll Surg. 2010;210:934-41.

89. Fahy BN, Fischer CP. Synchronous resection of colorectal primary and hepatic metastasis. J Gastrointest Oncol. 2012;3:48 58.

90. Grundmann RT. Current state of surgical treatment of liver metastases from colorectal cancer. World J Gastrointest Surg. 2011;3:183-96.

91. De Haas RJ, Adam R, Wicherts DA, Azoulay D, Bismuth H, Vibert E, et al. Comparison of simultaneous or delayed liver surgery for limited synchronous colorectal metastases. Br J Surg. 2010;97:1279-89.

92. Tsoulfas G, Pramateftakis MG. Management of rectal cancer and liver metastatic disease: which comes first? Int J Surg Oncol. 2012;2012:196908. doi: 10.1155/2012/196908

93. Mentha G, Roth AD, Terraz S, Giostra E, Gervaz P, Andres A, et al. 'Liver first' approach in the treatment of colorectal cancer with synchronous liver metastases. Dig Surg. 2008;25:430-5.

94. Chua TC, Saxena A, Liauw W, Kokandi A, Morris DL. Systematic review of randomized and nonrandomized trials of the clinical response and outcomes of neoadjuvant systemic chemotherapy for resectable colorectal liver metastases. Ann Surg Oncol. 2010;17:492-501.

95. Adam R, De Gramont A, Figueras J, Guthrie A, Kokudo N, Kunstlinger $\mathrm{F}$, et al. The oncosurgery approach to managing liver metastases from colorectal cancer: A multidisciplinary international consensus. Oncologist. 2012;17:1225-39. 
96. Grundmann RT, Hermanek P, Merkel S, Germer CT, Grundmann RT, Hauss J, et al. Diagnosis and treatment of colorectal liver metastases - workflow. Zentralbl Chir. 2008;133:267-84.

97. Khan K, Wale A, Brown G, Chau I. Colorectal cancer with liver metastases: Neoadjuvant chemotherapy, surgical resection first or palliation alone? World J Gastroenterol. 2014;20:12391-406.

98. Benoist S, Brouquet A, Penna C, Julié C, El Hajjam M, Chagnon $\mathrm{S}$, et al. Complete response of colorectal liver metastases after chemotherapy: Does it mean cure? J Clin Oncol. 2006;24:393945 .

99. Brouquet A, Mortenson M, Vauthey JN, Rodriguez-Bigas MA, Overman MJ, Chang GJ, et al. Surgical strategies for synchronous colorectal liver metastases in 156 consecutive patients: Classic, combined or reverse strategy? Am Coll Surg. 2010;210:6.

100. Pérez-Cabrera B, Palomeque-Jiménez A, Navarro-Sánchez P, González-Ramírez AR, Navarro-Freire F. Metástasis hepáticas de origen colorrectal sincrónicas: ¿intervención simultánea o secuencial? Rev Chil Cir. 2015:67:158-66.
101. Bajpai SK, Sahani D. Recent progress in imaging of colorectal cancer liver metastases. Curr Colorectal Cancer Rep. 2009;5:99-107.

102. Mainenti PP, Cirillo LC, Camera L, Persico F, Cantalupo T, Pace L, et al. Accuracy of single phase contrast enhanced multidetector CT colonography in the preoperative staging of colo-rectal cancer. Eur J Radiol. 2006;60:453-9.

103. Mainenti PP, Romano F, Pizzuti L, Segreto S, Storto G, Mannelli L, Imbriaco $\mathrm{M}$, et al. Non-invasive diagnostic imaging of colorectal liver Metastases. World J Radiol. 2015;7:157-69.

\section{Correspondencia: Camilo Naranjo, MD Correo electrónico: canasa89@gmail.com Medellín, Colombia}

